\title{
El Subsistema de Información sobre Nacimientos. Estudio de caso en una región indígena de Chiapas, México
}

\section{The Subsystem of Information on Births. Case study of an indigenous region of Chiapas, Mexico}

\author{
Graciela Freyermuth Enciso* \\ María del Pilar Ochoa Torres** \\ José Alberto Muños Hernández***
}

\section{Resumen}

El Subsistema de Información sobre Nacimientos (Sinac) de la Secretaría de Salud en México se ha convertido en una herramienta imprescindible para el análisis de la salud materno-infantil. Este subsistema se genera a través de los certificados de nacimiento (CN), y a partir del año 2011 fue propuesto como insumo para estimar la razón de mortalidad materna en México. Sin embargo, a la fecha pocos estudios muestran indicios sobre la confiabilidad de los datos del Sinac. Por tanto, el objetivo de esta investigación fue identificar la consistencia de la información de nacimientos en Chiapas, partiendo de un análisis de la base de datos y a través de un estudio de caso. El análisis permitió describir y analizar los obstáculos en la expedición oportuna del CN en la región Altos Tsotsil-Tseltal de Chiapas, donde gran parte de las mujeres continúa pariendo en casa. Los resultados muestran la necesidad de hacer modificaciones en el CN para su correcto llenado y su expedición oportuna. También muestra las dificultades que representa para las mujeres chiapanecas realizar el trámite del CN.

Palabras clave: Subsistema de Información sobre Nacimientos; certificado de nacimiento; registro civil; indígenas; inconsistencias; Chiapas; razón de mortalidad materna; subregistro; parto.

* Profesora investigadora del Centro de Investigaciones y Estudios Superiores en Antropología Social (CIESAS), unidad Sureste. Dirección postal: San Juan Chamula, Km 3.5, Barrio Quinta San Martín, 29247, San Cristóbal de Las Casas, Chiapas, México. Correo electrónico: gracielafreyermuth54@hotmail.com

** Asesora en la Subsecretaría de Integración y Desarrollo del Sector Salud. Dirección postal: Lieja 7, piso 1, col. Juárez, 06600, Ciudad de México, México. Correo electrónico: pilarochoatorres@gmail.com

*** Catedrático Conacyt-CIESAS, Pacífico Sur. Correo electrónico: kasteblok@yahoo. com.mx 


\begin{abstract}
The Birth Information Subsystem (Sinac) of the Mexican Ministry of Health has become an essential tool for the analysis of maternal and child health. This subsystem is generated through birth certificates (BC), and in 2011 it was proposed as an input to estimate the maternal mortality rate in Mexico. However, to date, very few studies have provided evidence of the reliability of Sinac data. The purpose of this research was therefore to identify the consistency of the information on births in Chiapas, based on an analysis of the database, and through a case study. The analysis made it possible to describe and analyze the obstacles in the timely issue of BCs in the Altos Tsotsil-Tseltal region of Chiapas, where the majority of women continue to give birth at home. The results show the need to modify BCs to ensure that they are correctly filled in and issued in a timely manner. They also show the difficulty that Chiapas women experience in completing the procedure to obtain their children's $B C$.
\end{abstract}

Keywords: Birth Subsystem Information; birth certificate; civil registration; indigenous peoples; inconsistencies; Chiapas; maternal mortality ratio; underreporting; birth.

\title{
Introducción
}

El análisis de la composición poblacional constituye un elemento importante para la generación y organización de los datos demográficos y sociales, los cuales se utilizan para la creación de planes, políticas o programas encaminados a resolver problemas poblacionales (INEGI, 2003). En México se cuenta con una importante tradición de estadísticas vitales. Desde la segunda mitad del siglo XIX aparecieron los primeros registros civiles (RC) del país y posteriormente, a fines del mismo siglo, el censo de población, los cuales permitieron el estudio de la mortalidad y los nacimientos (Valdez, 2009).

Sin embargo, la calidad de los datos podría considerarse deficiente hasta antes de 1940 (Camposortega, 1992: 16). Es entre 1948 y 1951 cuando se publicó un decreto presidencial que establecía que se debía adoptar el modelo de certificado de defunción que proponía la Organización Mundial de la Salud (OMS), y a partir de entonces se empezó a utilizar un formato que, además de los datos de la persona que falleció, incluía una sección médica que permitía anotar las causas de muerte, complicaciones y causas contribuyentes (Lozano, 2008). En 1984 apareció la Ley General de Salud, y con ella la atribución de la Secretaría de Salud (SSA) para aprobar los certificados de defunción; dos años después se expidió un decreto en el Diario Oficial de la Federación (DOF) donde se normaba la expedición de este tipo de certificados (Lozano, 2008: 526). 
Al igual que en otros países subdesarrollados, en México el registro de los nacimientos y las defunciones todavía presenta problemas de confiabilidad (González y Cárdenas, 2005), observándose distintos tipos de errores como omisiones o equivocaciones y el registro tardío. Para solventar los problemas del registro de nacimientos se han desarrollado distintos métodos de análisis que hacen posible una estimación de parámetros de estos eventos, sobre todo a través de encuestas, para de esa forma reemplazar al método tradicional, que es el registro civil. Al mismo tiempo, estos métodos son útiles para evaluar la calidad de la información (Aguirre y Camposortega, 1980; Welti, 2009).

La instauración del certificado de nacimientos a través de la Dirección General de Información en Salud (DGIS) está encaminada a remontar el problema de cobertura y oportunidad del registro del recién nacido. Sin embargo, a pesar de que desde 2008 comenzó su expedición, la DGIS ha señalado que a la fecha son nueve los estados que requieren corrección del subregistro de nacidos vivos: Chiapas, Chihuahua, Guerrero, Hidalgo, Oaxaca, San Luis Potosí, Sonora, Veracruz y Yucatán. En el caso específico de Chiapas, al comparar los nacimientos estimados en 2013 por el Consejo Nacional de Población (Conapo) y los captados por los Certificados de Nacimiento $(\mathrm{CN})$, la diferencia de los estimados (111 733) y los certificados (91 189) es de 18\%. Los contrastes más grandes entre estos dos grupos ocurren en municipios con pocos nacimientos, como los casos de Chicoasén con 125 certificados y 85 estimados, es decir, una diferencia de $48 \%$; o el caso de San Andrés Duraznal con 7 certificados y 180 estimados, con una diferencia de -96 por ciento.

Por tanto, considerando la importancia del certificado de nacimiento, el objetivo del estudio es analizar la confiabilidad de los datos del Subsistema de Información sobre Nacimientos (Sinac) para el caso de Chiapas, y describir la forma en que se llenan los $\mathrm{CN}$ en la región Altos Tsotsil-Tseltal de Chiapas (en adelante Los Altos), zona donde las mujeres suelen atender sus partos en casa. Lo anterior a partir de dos objetivos específicos: sistematizar y analizar las bases de datos del Sinac para el estado de Chiapas, con la finalidad de identificar inconsistencias en el registro de información y averiguar si algunas variables se encontraban mal registradas; adicionalmente, realizar un análisis etnográfico institucional en la región Los Altos para establecer, a través de los actores clave del Sistema Nacional de Salud (SNS) y de oficialías del RC, cuáles son los problemas para el registro y llenado del CN. 


\section{Antecedentes}

Según la Secretaría de Salud de México, el certificado de nacimiento

[...] es un documento oficial de carácter individual e intransferible que certifica el nacimiento de un producto vivo en el momento mismo de su ocurrencia, proporcionando a la madre un comprobante de este hecho. Es un formato único de registro de nacimientos de uso obligatorio en el territorio nacional [http:// www.dgis.salud.gob.mx/contenidos/difusion/cnacimiento.html, 2016].

Los antecedentes del $\mathrm{CN}$ como documento imperativo son recientes: el 2 de mayo de 2007 se firmaron, por parte de la Secretaría de Gobernación y la SSA, las Bases de Colaboración para el Uso del CN, en las que se establece como obligatoria la presentación del $\mathrm{CN}$ original para la obtención del acta de nacimiento correspondiente. Por tanto, el $\mathrm{CN}$ quedó suscrito como documento obligatorio y gratuito.

La DGIS, con base en lo establecido en el Reglamento Interior de la SSA (DOF, 2 de febrero de 2010, citado en SSA-DGIS, 2010: 17), es la instancia responsable de establecer el diseño y contenido de los certificados. A su vez, tal dirección generó el Sinac, que concentra la información captada por el CN; el subsistema forma parte del Sistema Nacional de Información en Salud en México (Sinais), cuyo principal objetivo es captar, producir y difundir información de recursos físicos, materiales, humanos y financieros, de servicios otorgados, de morbilidad y mortalidad hospitalarias, así como de mortalidad general.

En los dos primeros años de la puesta en marcha del CN (2008-2009), el Sinac logró una cobertura mayor al 100\% de las proyecciones realizadas por el Conapo para esos años (SSA-DGIS, 2010: 21); con base en ello, la DGIS señala que los $\mathrm{CN}$ permitirán contar con información oportuna y confiable sobre un indicador que es utilizado en el cálculo de las tasas de fecundidad y natalidad, además de que desde el año 2011 se utiliza la información del Sinac para la estimación de la RMM en México.

Los estudios acerca de la fiabilidad de la información de los certificados en nuestro país se centran en la mortalidad. Investigaciones previas del periodo 1940-1960 se enfocaron en el análisis del subregistro de la mortalidad infantil mediante el método etnográfico, analizando actas de defunción del RC y comparándolas con informes de las unidades médicas. Dichas investigaciones obtuvieron resultados semejantes y concluyeron que las tasas de mortalidad infantil estimadas a partir de los datos del RC tuvieron un subregistro de 30\% en promedio (Ordorica y cols., 1975; Aguirre y Camposortega, 1980; Suárez, 1981). 
Un estudio realizado en la Ciudad de México con una muestra de 574 defunciones perinatales mostró que $22 \%$ no fueron inscritas en el RC (Bobadilla y cols., 1987); en el mismo sentido, en un análisis realizado en Lima, Perú, también se encontraron discrepancias sobre la causa de muerte entre el RC y los registros hospitalarios (Espíritu y cols. 2007). En Guerrero el subregistro de muertes infantiles en 1994 fue de 73\% para las localidades de menos de 500 habitantes, y en las de más de 500 habitantes fue de $60 \%$, encontrándose como características asociadas al subregistro el carecer de certificados de defunción, desconocer las funciones del RC, la lejanía del $\mathrm{RC}$, acceso a un servicio médico a más de 30 minutos y ocurrencia de la defunción en localidades menores a 1000 habitantes (Tomé y cols., 1997). En otro análisis, Colón comparó las causas de muerte perinatal observadas en las actas de defunción del RC de 1995 a 1996 y encontró que, a partir de capacitaciones sobre reanimación neonatal, se redujo este tipo de mortalidad en un año (Colón y cols., 1998). Por otro lado, un estudio realizado con una muestra de 20 municipios de muy bajo índice de desarrollo humano en siete estados de la República Mexicana, reportó un subregistro de defunciones infantiles de 22.3\% entre 2007 y 2008 (Hernández y cols., 2012).

Otro grupo de investigaciones se ha centrado en el registro de la mortalidad materna (MM). En 2003 la Dirección General de Información en Salud (DGIS) realizó un estudio a través de los certificados de defunción, expedientes o resúmenes clínicos, los resultados de necropsias, los dictámenes de mortalidad, las aclaraciones de los certificantes y la realización de autopsias verbales (Lozano et al., 2005). Esta investigación documentó que aún prevalecía un mal registro de muertes maternas en el país, estimando un error de $17 \%$ en 2003 y de 12\% en 2004 (SSA-DGIS, 2009: 19). En el caso de Los Altos de Chiapas, para 2001 el subregistro fue de $70 \%$ con respecto a la información de las actas de defunción, y de $63 \%$ con respecto a los certificados de defunción (Freyermuth y Cárdenas, 2009); para ciertas comunidades mayas del oriente de Yucatán, entre 1998 y 1999 el subregistro fue de 40 y $50 \%$ respectivamente (Rodríguez-Angulo y cols., 2009).

Lo que se espera de los nacimientos es que su registro sea universal y oportuno, ya que es una fuente fundamental para estimar no sólo tasas de fecundidad, sino la mortalidad infantil y la RMM. Sin embargo, el primer problema que presenta este registro es el plazo que la población tiene para realizar este trámite, de hasta 180 días. Beatriz Figueroa (2009) mostró que la solicitud de un acta de nacimiento responde más a las necesidades particulares del ciclo de vida de las personas, como obtener la cartilla de vacunación o inclusive tener la oportunidad de beneficiarse de los programas de desarrollo social como sesenta y más. 
Un estudio sobre la cobertura oportuna y tardía de los registros de nacimientos ocurridos durante 1999 y 2009 encontró que en 1999 hubo un subregistro de $5.8 \%$ de niños menores de cinco años, teniendo las mayores cifras de subregistro los estados de Quintana Roo (20\%), Chiapas (17\%), Morelos (13\%) y Guerrero (9.1\%). El panorama para 2009 fue diferente ya que se dieron iniciativas que contribuyeron a disminuir el subregistro, como la obligatoriedad de la educación preescolar desde los tres años (UNICEF e INEGI, 2012). El estudio coincide con un análisis realizado con datos de 2014 en el que se encontró que el estado de Chiapas era el que contaba con un menor porcentaje de registros de niños de 0 a 5 años (90\%), en contraste con Querétaro, el cual tenía la cifra más alta, de 98.7\% (García, 2016).

\section{Material y métodos}

Se realizó un análisis de la base de datos de nacimientos del Sinac de $2013^{1}$ con la finalidad de verificar la calidad de la información, en términos de su cobertura y contenido. Debido a que los datos provienen de estadísticas vitales, para verificar la calidad se realizaron cálculos de frecuencias y diferencias relativas de las variables, así como cruce de las variables que están relacionadas y que conforman el CN. Se calculó la edad a partir de la fecha de nacimiento de la madre, y se elaboraron tablas de contingencia entre las siguientes variables: edad, escolaridad, ocupación de la madre, ¿hijo anterior nació...?, vive aún hijo anterior, número de embarazos, fecha de nacimiento del hijo del embarazo anterior y orden de nacimiento. Se reconocieron inconsistencias y/o valores atípicos ${ }^{2}$ que posiblemente son resultado de un inadecuado registro u omisión de los datos solicitados en el $\mathrm{CN}$. A las variables también las llamaremos ítems para hacer referencia a su acomodo numérico en el formato del $\mathrm{CN}$.

La importancia de revisar la calidad de la información, entre otras cosas, radica en el hecho de que sin datos sobre la edad de las madres, el orden de los nacimientos o las características socioeconómicas de los padres, no es posible conocer los patrones reproductivos de la población (CEPAL, 2014). Para identificar la ausencia de información, se revisaron los registros que en sus opciones de respuesta contuvieran: No Especificado (NE), o Se Ignora (SI). La posibilidad de un alto porcentaje de NE permite reconocer problemas en el registro de datos por parte del o de la certificante; por otro lado, un alto

1 Disponible en: http://www.dgis.salud.gob.mx/contenidos/basesdedatos/std_nacimientos. html (15 de agosto, 2016).

2 Observación que es numéricamente distante del resto de los datos. 
porcentaje de SI podría suponer que la o el informante no es adecuado y desconoce la respuesta, o que las preguntas del instrumento no son comprendidas cabalmente. En este estudio se analizan aquellas variables que muestran rangos de NE o SI que sobrepasan 5\%, un parámetro bastante estricto. Esta primera parte del análisis se realizó con la información de todos los municipios del estado de Chiapas.

Para el análisis de los $\mathrm{CN}$ se incluyó la revisión de la calidad de la información municipal. Para ello una opción es comparar la información del Sinac con una fuente de información distinta (CEPAL, 2011). Sin embargo, se tiene la dificultad de conseguir información anual municipal. Por tanto, para la comparación se utilizaron las proyecciones de población que publicó el Conapo para el periodo 2010-2018, considerando a éstas como un proxy de los nacimientos que idealmente deberían estarse registrando.

También se llevó a cabo trabajo de campo en unidades de atención del Instituto de Salud de Chiapas (ISECH), encargadas de la expedición de certificados. El trabajo de campo se realizó de julio a septiembre de 2014 en diez unidades de salud de la región Los Altos, en donde se entrevistó a los encargados de llenar los CN. Asimismo se interrogó a los responsables del registro de esta información en tres hospitales; también se mantuvieron conversaciones con algunos responsables jurisdiccionales (Ocosingo, Palenque y San Cristóbal) y se entrevistó a los responsables de las oficialías de $\mathrm{RC}$ de 14 municipios de la región.

Esta área geográfica (constituida por 17 municipios, Mapa 1) fue elegida debido a que en la mayoría de sus municipios (15) prácticamente toda la población es hablante de alguna lengua indígena (98\%) y 54\% no habla español -es decir, es monolingüe-(INEGI, 2010). La ciudad más importante de esta región y punto de confluencia de estos municipios es San Cristóbal de Las Casas, en la que $36.7 \%$ de la población es indígena y $7 \%$ monolingüe.

Esta región ha sido foco de atención tanto de los gobiernos federales como estatales en los últimos diez años a fin de disminuir la MM. En 2012 el 16\% de las MM en Chiapas ocurrieron en la región Los Altos, el mayor porcentaje de las 15 regiones del estado. La RMM en la entidad fue de 83, casi el doble del promedio nacional.

En la última década hubo un incremento importante de los servicios de salud. Para 2014 existían dos hospitales del ISECH - uno materno infantil y uno general-y un hospital rural de IMSS-Prospera. De acuerdo con información oficial hay cinco hospitales comunitarios en la región, establecidos en los 18 municipios que la conforman.

Para 2013 se estimaron por Conapo 17186 nacimientos en Los Altos, y a través de Sinac 7558 , es decir, existió una diferencia de 44\%; las dife- 


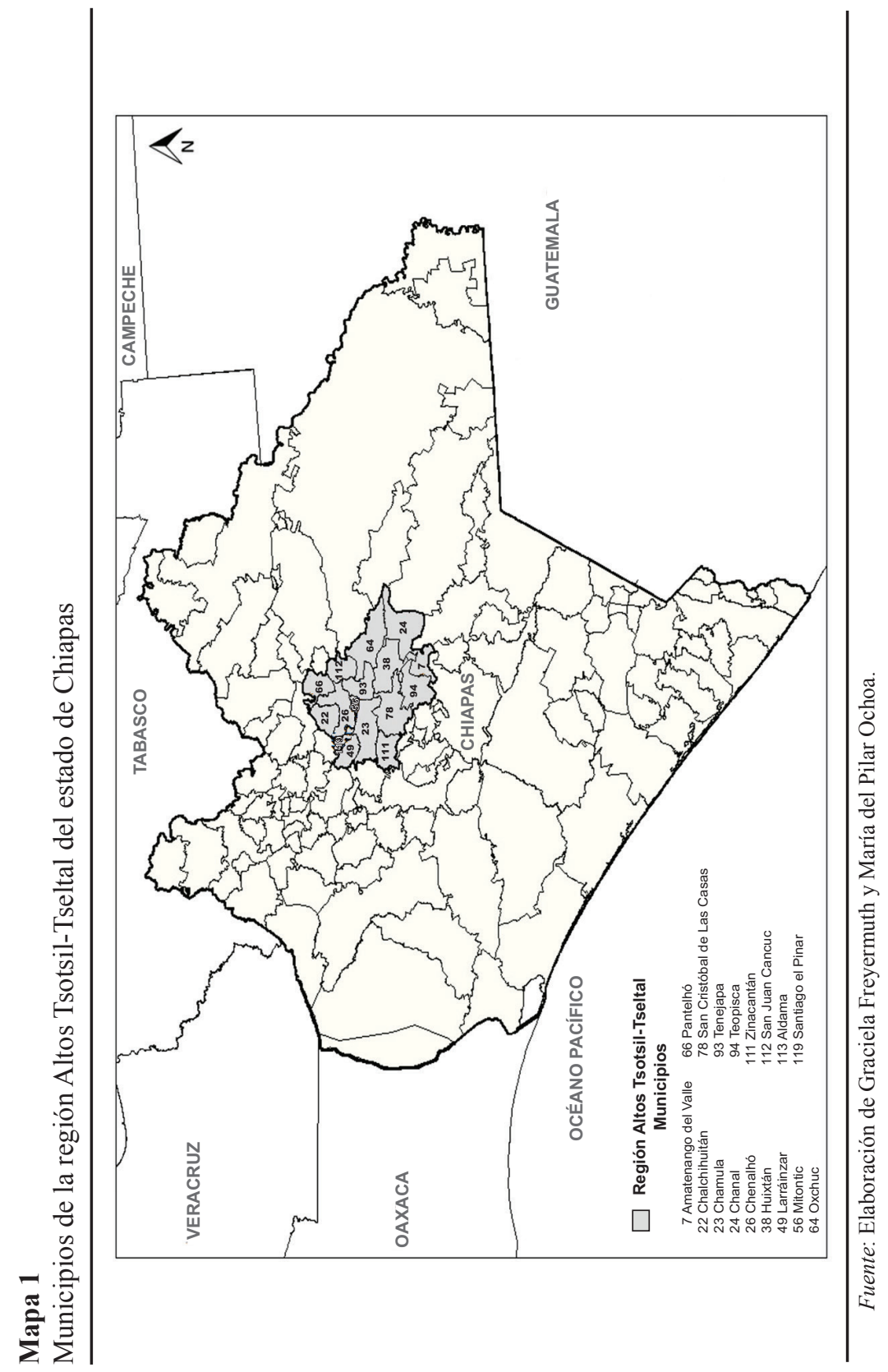


rencias en cada municipio fueron de $10 \%$ en la ciudad de San Cristóbal de Las Casas, y hasta 95\% para el caso de Santiago El Pinar (Cuadro 1).

Estas diferencias pueden deberse a los usos y costumbres entre los tsotsiles y tseltales, ya que a los niños y niñas muy pequeñas no se les asigna nombre porque se piensa que a esa edad el alma puede separarse fácilmente del cuerpo. Con el fin de proteger a su alma, el o la menor no es presentada ante extraños en los primeros meses de su vida (Guiteras, 1992; De León, 1999). Sin embargo, para los y las niñas que han nacido en otro contexto social -en la ciudad de San Cristóbal, por ejemplo- se modifican

\section{Cuadro 1}

Diferencias porcentuales entre el número de certificados captados por el Sinac 2013 y RN estimados a través de proyecciones del Conapo 2010-2018, según municipios de la región Altos Tsotsil-Tseltal, Jurisdicción Sanitaria II, Chiapas, 2013

\begin{tabular}{|c|c|c|c|}
\hline Municipio & Sinac 2013 & $\begin{array}{c}\text { Estimados } \\
\text { por el Conapo, } \\
2010-2018 \\
\end{array}$ & $\begin{array}{l}\text { Porcentajes } \\
\text { de diferencia }\end{array}$ \\
\hline Amatenango del Valle & 70 & 229 & 69 \\
\hline Chalchihuitán & 72 & 472 & 85 \\
\hline Chamula & 192 & 2558 & 92 \\
\hline Chanal & 51 & 346 & 85 \\
\hline Chenalhó & 107 & 1116 & 90 \\
\hline Huixtan & 244 & 512 & 52 \\
\hline Larráinzar & 596 & 667 & 11 \\
\hline Mitontic & 107 & 420 & 75 \\
\hline Oxchuc & 758 & 1112 & 32 \\
\hline Pantelhó & 115 & 647 & 82 \\
\hline Las Rosas & 436 & 483 & 10 \\
\hline San Cristóbal de Las Casas & 3800 & 4254 & 11 \\
\hline Tenejapa & 156 & 1173 & 87 \\
\hline Teopisca & 348 & 961 & 64 \\
\hline Zinacantán & 160 & 1098 & 85 \\
\hline San Juan Cancuc & 222 & 887 & 75 \\
\hline Aldama & 120 & 170 & 29 \\
\hline Santiago el Pinar & 4 & 81 & 95 \\
\hline Totales & 7558 & 17186 & 44 \\
\hline
\end{tabular}

Fuente: Elaboración de Graciela Freyermuth, a partir de Cubos dinámicos. Proyecciones de la población municipal de México, 2010-2018 (SSA-DGIS, varios años), Conapo (varios años) y Certificado de Nacimiento (SSA-Sinac, 2013). 
estas prácticas y representaciones en la medida en que las madres comerciantes deben cargar con ellos desde muy pequeños en lugares públicos que comparten con los mestizos. Es decir, estas concepciones en torno a los riesgos que puede sufrir un grupo generacional en particular, son modeladas o modificadas por otras relaciones sociales.

\section{Resultados}

En este apartado se presenta el análisis de la calidad de la información que contiene la base de datos del Sinac, y se detalla la forma en que se asientan los $\mathrm{CN}$ en Los Altos en las unidades médicas. El objetivo es mostrar los problemas que se tienen, en este complejo contexto, para la certificación oportuna de los nacimientos.

El análisis de la información del Sinac se describe en tres apartados: "Datos de la madre", con información sociodemográfica que aparece en el $\mathrm{CN}$ en los ítems 14 al 16, los cuales se presentan primero ya que son los datos de identificación; siguiendo con "Historia reproductiva de la madre"3 (ítems 6 al 11.3), y finalmente "Datos del nacido vivo y del nacimiento" (ítems 16 al 29.5).

\section{Análisis de los datos del Sinac}

En el Cuadro 2 se exponen el número de municipios en los que la respuesta NE o SI sobrepasó 5\%, y el rango con los valores mínimo y máximo observados para el total de los municipios de Chiapas según cada variable.

Por un lado, en 14 de las 32 variables incluidas en el CN hubo NE de más de 5\%, encontrándose irregularidades con NE y SI en 113 municipios. Para la información con NE el menor rango fue para las variables de persona que atendió el parto (0-6\%) y vacuna $B C G(0-7 \%)$ en uno y cinco municipios respectivamente. Le siguen la edad de la madre y el número de hijos nacidos muertos $(0-10 \%)$ en cinco y seis municipios en cada caso. Los mayores intervalos sobrepasan el $80 \%$ para las variables edad gestacional $(0-82 \%)$ en diez municipios, Apgar (0-83\%) y Silverman (0-88\%) en 31 municipios, y anomalías congénitas, enfermedades o lesiones del nacido vivo $(0-87 \%)$ en 53 municipios.

3 En el CN éste forma parte del apartado "Datos de la madre", pero en la descripción las separamos para tratar sólo el historial reproductivo, sin datos de identificación. 
Por otro lado, encontramos que 18 variables presentan datos con menos de $5 \%$ de mal registro y casi todas éstas corresponden a datos de la madre. La información que presenta un rango mayor de no respuesta es sobre el recién nacido $(\mathrm{RN})$ y la historia reproductiva de la madre.

En cuanto a los resultados de la columna correspondiente a SI, de las 32 variables incluidas en el $\mathrm{CN}$, hay 13 en las que hubo un mayor rango de ausencia de información que incluyen a la variable ¿hijo anterior nació...? (0-27\%) en 31 municipios, vacuna BCG (0-61\%) en 43 municipios, hepatitis $B(0-61 \%)$ en 43 municipios, vitamina $A(0-62 \%)$ en 46 municipios, tamiz neonatal auditivo (0-63\%) en 53 municipios, y afiliación a servicios de salud (0-66\%) en 23 municipios.

\section{Datos de la madre}

Los datos de identificación de la madre como lugar de nacimiento y residencia habitual están adecuadamente registrados, pero se encontraron inconsistencias de más de $5 \%$ en la fecha de nacimiento en cinco municipios con $\mathrm{NE}$ hasta en 10 por ciento.

Es importante señalar que en el cruce de las variables de datos de la madre con los de la historia reproductiva, las inconsistencias en ningún caso sobrepasaron $5 \%$. Sin embargo, este ejercicio puede servir para identificar aspectos problemáticos en el llenado del $\mathrm{CN}$ y mejorar las instrucciones de registro, e incluso para incluir estos aspectos durante la capacitación para el llenado del CN. Es importante documentarlas ya que nos sugiere que pueden estar mal capturadas, aunque esto no sea evidente.

En las menores de 24 años la escolaridad no corresponde con la edad, por ejemplo: Profesional y Posgrado en jóvenes de 12 a 16 años que debieran tener bachillerato a lo sumo. Para evitar interpretaciones del certificante o del informante sería deseable explicitar años de estudio, o señalarse en el Manual para el llenado del certificado de nacimiento quién puede ser considerada como una profesionista o con estudios de posgrado.

Estas inconsistencias se observan en Profesionistas y Trabajadoras de la Educación con edades de 16 años. Esta última categoría es posible en el contexto rural de Chiapas, y también es probable que se estén considerando como profesionistas a aquellas que tienen una capacitación técnica. Estos datos de la madre deberían formar parte de sus datos sociodemográficos (Figura 1). 


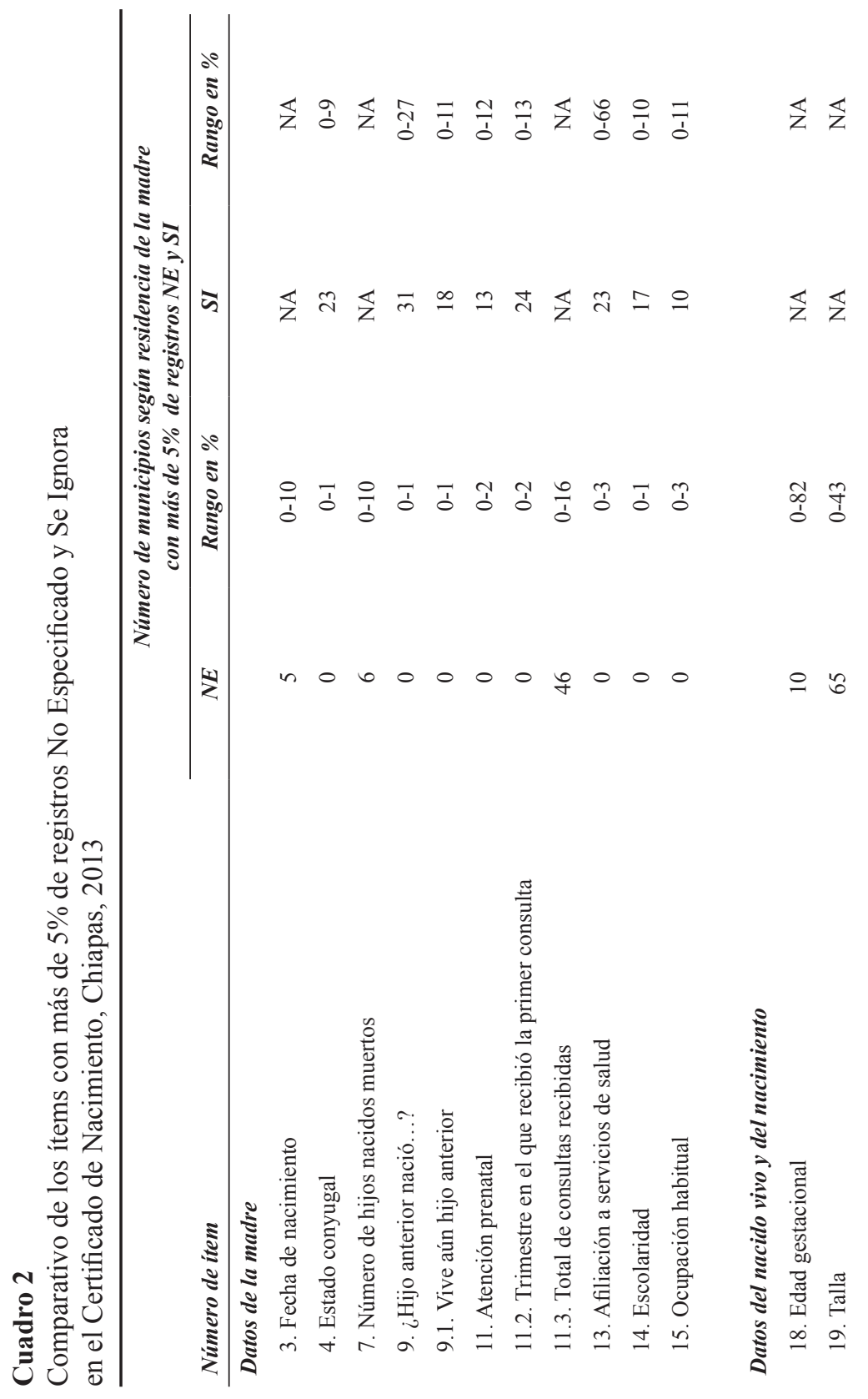




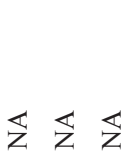

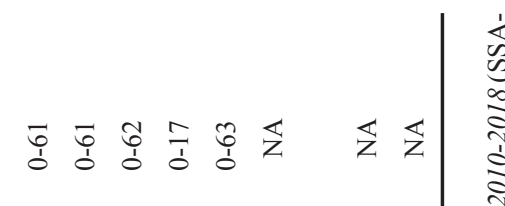

$$
\begin{aligned}
& \overleftrightarrow{z} \overleftrightarrow{z} \\
& \text { ช์ำ } \\
& \text { 象爱 }
\end{aligned}
$$

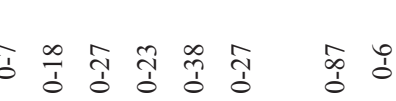

$$
\begin{aligned}
& \cong \bar{m} \bar{m} \\
& n \infty a \sigma \pm 0
\end{aligned}
$$

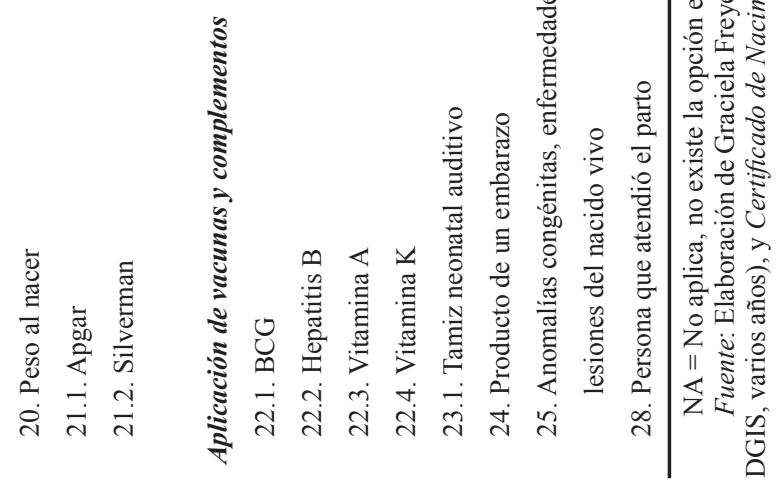

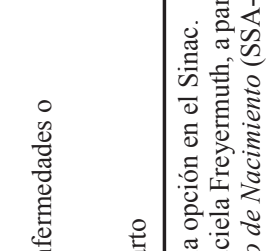




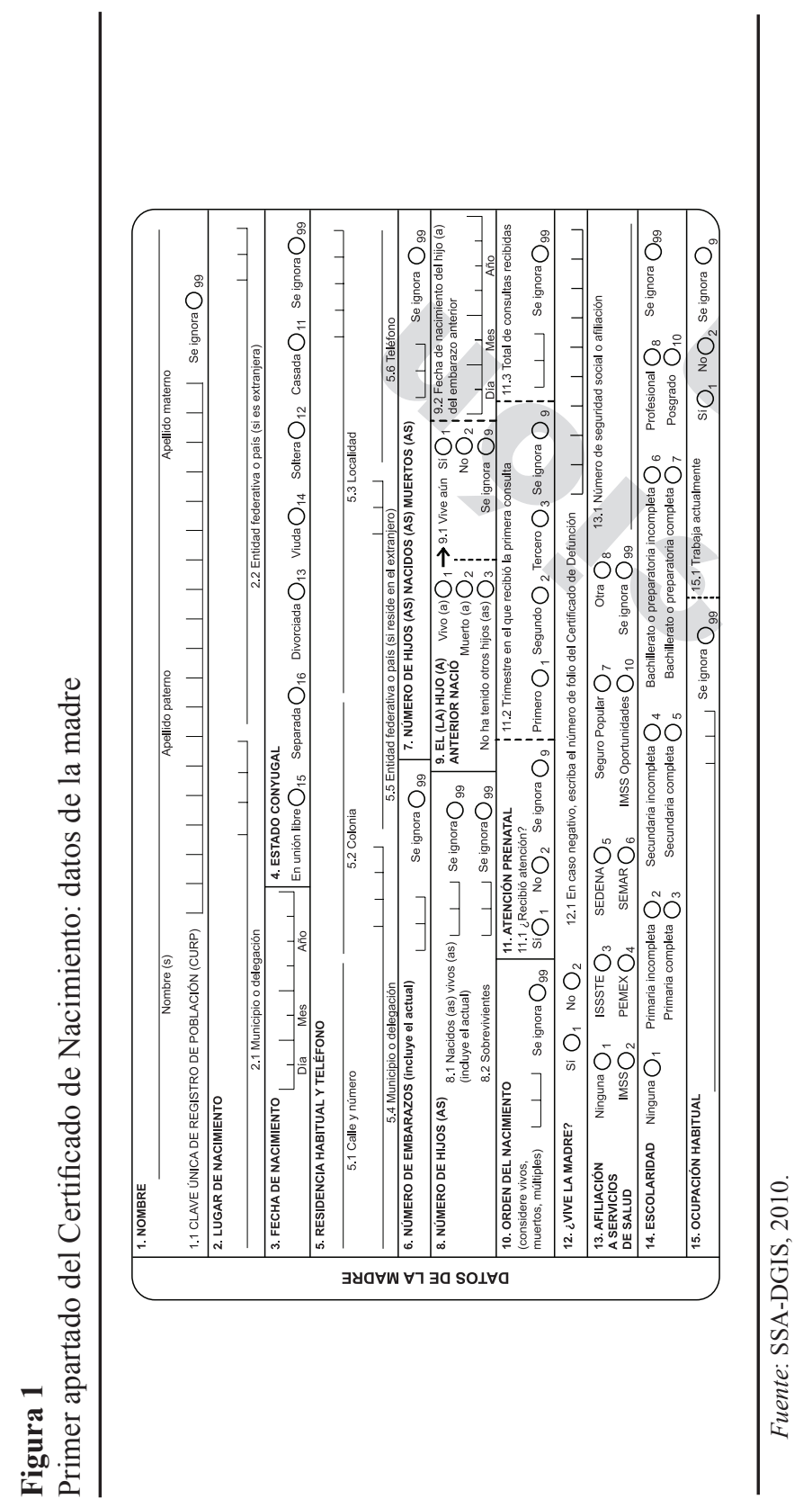




\section{Historia reproductiva de la madre}

Incluye el número de hijos(as), número de embarazos y número de hijos(as) nacidos(as) muertos(as). No se cuenta con un ítem que indague sobre el número de abortos y por ello es difícil la validación de la historia reproductiva, aunque en las especificaciones se establece que se considera entre los nacidos muertos al aborto y a la mola hidatiforme; en sentido estricto los abortos no serían nacidos muertos, ya que para estos últimos la posibilidad de estar vivos antes de nacer es nula. Entonces, incluir la historia reproductiva sin todos sus componentes posiblemente conlleve problemas en el registro. Lo anterior, dado que la suma de nacidos vivos y muertos -contemplando a los partos únicos o múltiples-, no corresponde con el número de embarazos. Es posible que, aunque esté especificado en las instrucciones, si este instrumento es registrado por personal de salud, no se esté considerando a un aborto como un nacido muerto.

La fecha de nacimiento del hijo(a) del embarazo anterior es una variable que presenta inconsistencia si se relaciona con el número de embarazos, hijos nacidos vivos, muertos, y sobrevivientes. Es así que su análisis debe realizarse con cautela.

Consideramos que los errores en el registro de las variables el(la) hijo(a) anterior nació..., vive aún, atención prenatal y trimestre en el que recibió la primera consulta, están relacionados con el informante (SI) más que con el certificante (NE); esto puede estar ocurriendo porque en Chiapas un porcentaje de mujeres es monolingüe y los informantes son hablantes de español, por lo que no hay comunicación efectiva entre ambos y puede no comprenderse el significado de la pregunta.

¿Vive la madre? Esta variable no es consistente con los resultados de la base de MM, ya que en 2013 ocurrieron 46 MM en las instituciones públicas y privadas, y 17 en otros lugares distintos a las unidades de salud (Freyermuth, Luna y Muños, 2015); gran parte de estas muertes ocurrieron en el posparto inmediato, sin embargo, por medio del CN solamente se registraron 11.

Cuando utilizamos las variables vive aún (hijo anterior) o la variable orden de nacimiento, pueden reconocerse inconsistencias, ya que si no hay hijo anterior o es el primer nacimiento no es posible que existan otros hijos vivos o muertos. Adicionalmente, es importante señalar que algunas de las variables como orden de nacimiento, hijos vivos, hijos muertos, datos sobre el hijo anterior (fecha, muerte o sobrevivencia), no se encuentran incluidas en otros formatos estandarizados que utiliza el Instituto de Salud del Estado de Chiapas (ISECH), como el formato de Egresos Hospitalarios y la Nota de Alta y de Contrarreferencia. Esto adquiere importancia durante el llenado de los $\mathrm{CN}$. 


\section{Datos del nacido vivo y del nacimiento}

En el segundo apartado se observa una mayor cantidad de municipios con porcentaje de NE superior a 5\% (Cuadro 2) y se identifica que en las preguntas 18 a 21 no se cuenta con la opción SI (Figura 2).

La captación correcta de los datos pertenecientes a este apartado depende de la temporalidad con la cual se capture la información. En algunos hospitales se levanta el certificado hasta que la madre ha egresado; para ello se le solicitan documentos de los cuales carece y debe regresar posteriormente con la certeza de que los procedimientos que se tienen contemplados se han realizado, por ejemplo, tamizaje y vacunación. Este apartado, en las valoraciones Apgar y Silverman y la aplicación de vitaminas y vacunas, tiene dificultades en su llenado. Es posible que esto ocurra porque la información referente a la aplicación de vitaminas y vacunación aparece en las notas de enfermería; pero en el caso de las inmunizaciones, no se especifica su tipo en el formato disponible. Así que, aunque se consulte la historia clínica, se desconoce cuáles vacunas fueron aplicadas. Sería importante identificar de manera más estrecha los problemas que tienen los responsables de la captura en torno a la aplicación de vitaminas y vacunas.

Es factible que en algunos centros de salud el certificado se llene inmediatamente después del nacimiento, aunque el trabajo de campo realizado durante esta investigación indica que eso no ocurre en la Jurisdicción II. La valoración de Silverman y la aplicación de vacunas y vitaminas no están incluidas en el formato Egresos Hospitalarios, ni tampoco en la Nota de Alta y de Contrarreferencia. Esta información debería conocerla cualquier mujer al ser dada de alta después de un parto.

Este apartado requiere de una estrategia particular, sobre todo para que las madres a su egreso o el personal de estadística cuenten con un documento que incluya estos datos. Cuando el certificado se hace en el hospital frecuentemente no se utiliza el expediente clínico para su llenado (hay registros con NE o SI que provienen de los servicios públicos del Sector Salud), por ello consideramos que debe haber un formato para todo el Sector Salud con el fin de que la madre egrese con esta información y pueda proporcionarla al momento del llenado del CN, lo que no siempre ocurre durante la estancia en el hospital. En el Manual no se especifica cómo se obtienen estos datos, aunque se señala que cuando el nacimiento ocurra en un servicio de salud no deben quedar sin registrar. Se tendría que especificar que el certificante deberá consultar el expediente clínico para el llenado del $\mathrm{CN}$, actividad que realiza en Palenque la encargada de los $\mathrm{CN}$. 


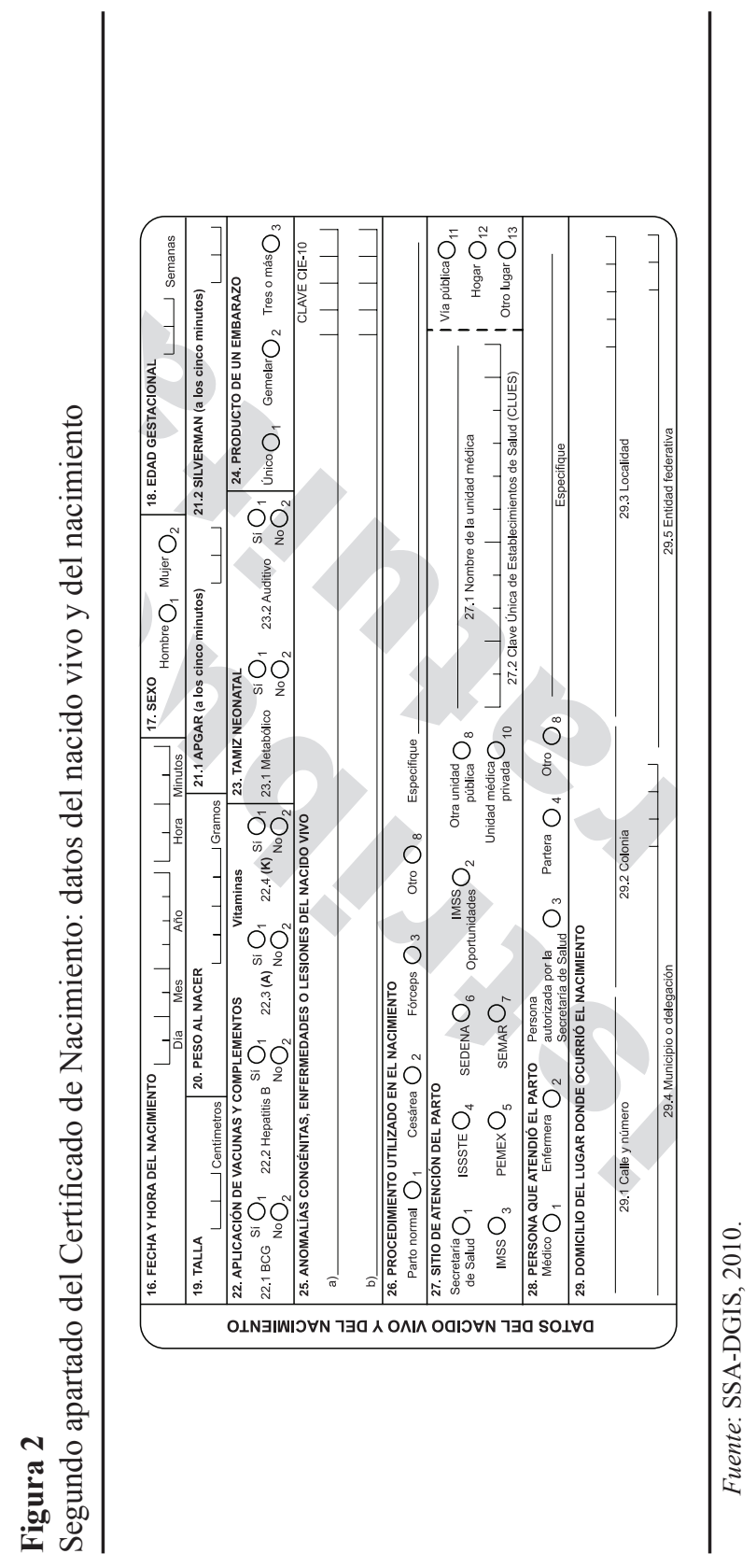


Los datos del RN que se certifican incluyen los ítems 24 a 29 (Figura 2). En producto de un embarazo, seis municipios contaron con respuestas $\mathrm{NE}$ en un rango de 0-27\%; anomalías congénitas, enfermedades o lesiones del nacido vivo se encuentra capturado a través de dos variables, principales causas $L M^{4}$ y clave $C I E,{ }^{5}$ sólo que en la primera, la respuesta asociada a NE es "las demás causas". En este ítem hay 53 municipios con rango de 5 a $85 \%$ de NE. En persona que atendió el parto se observa únicamente un municipio con $6 \%$ de respuesta NE. El procedimiento utilizado en el nacimiento y el sitio de atención del parto no tienen problemas de NE o SI. Los ítems 29 y 30 incluyen datos personales que no son accesibles en la base de datos y el siguiente apartado, el del certificante (ítems 31 a 36), tampoco tiene problemas de NE o SI (Figura 3).

Por otro lado, el no registro de variables también se observa en el CN de partos que ocurrieron tanto en las instituciones de salud como fuera de ellas. En el Cuadro 3 se muestran los porcentajes de NE y SI de algunas variables seleccionadas por lugar de ocurrencia del nacimiento. Se puede reconocer, en primer lugar, que en los partos institucionales no se registran al $100 \%$ datos como el peso del RN, o no se especifica si se realizó la aplicación de vacunas o vitaminas; sobre todo en el IMSS Oportunidades y la SSA el desconocimiento o SI es de alrededor de 5\% para cada una de las variables. En segundo lugar, se observa una elevada desinformación cuando no se ha especificado el sitio de atención del parto con los NE y SI de las variables. Esto apuntaría a que se tenía la intención de emitir un $\mathrm{CN}$ sin importar si el informante era el adecuado, pues valores elevados se esperarían con nacimientos en la vía pública, el hogar u otro sitio, como se observa en la información del Cuadro 3.

4 Principales causas con Lista Mexicana, disponible en los Cubos dinámicos del Sinac a partir de 2010.

5 Clasificación Internacional de Enfermedades. 


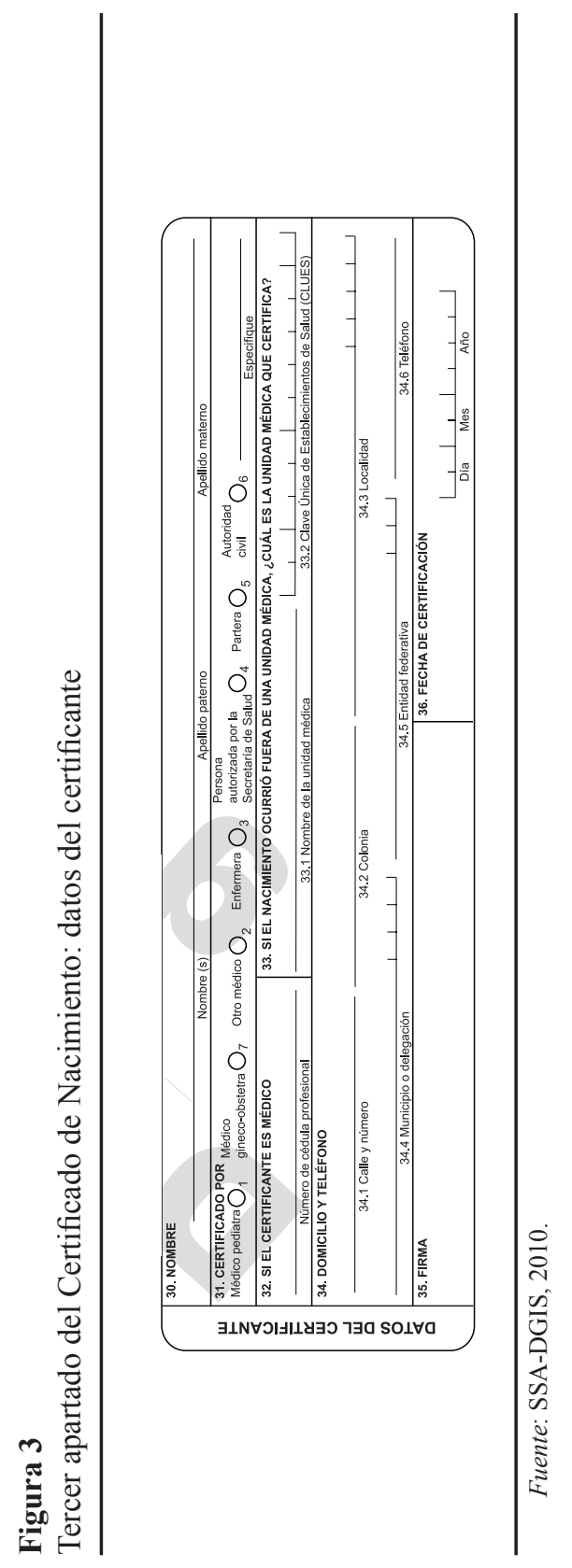




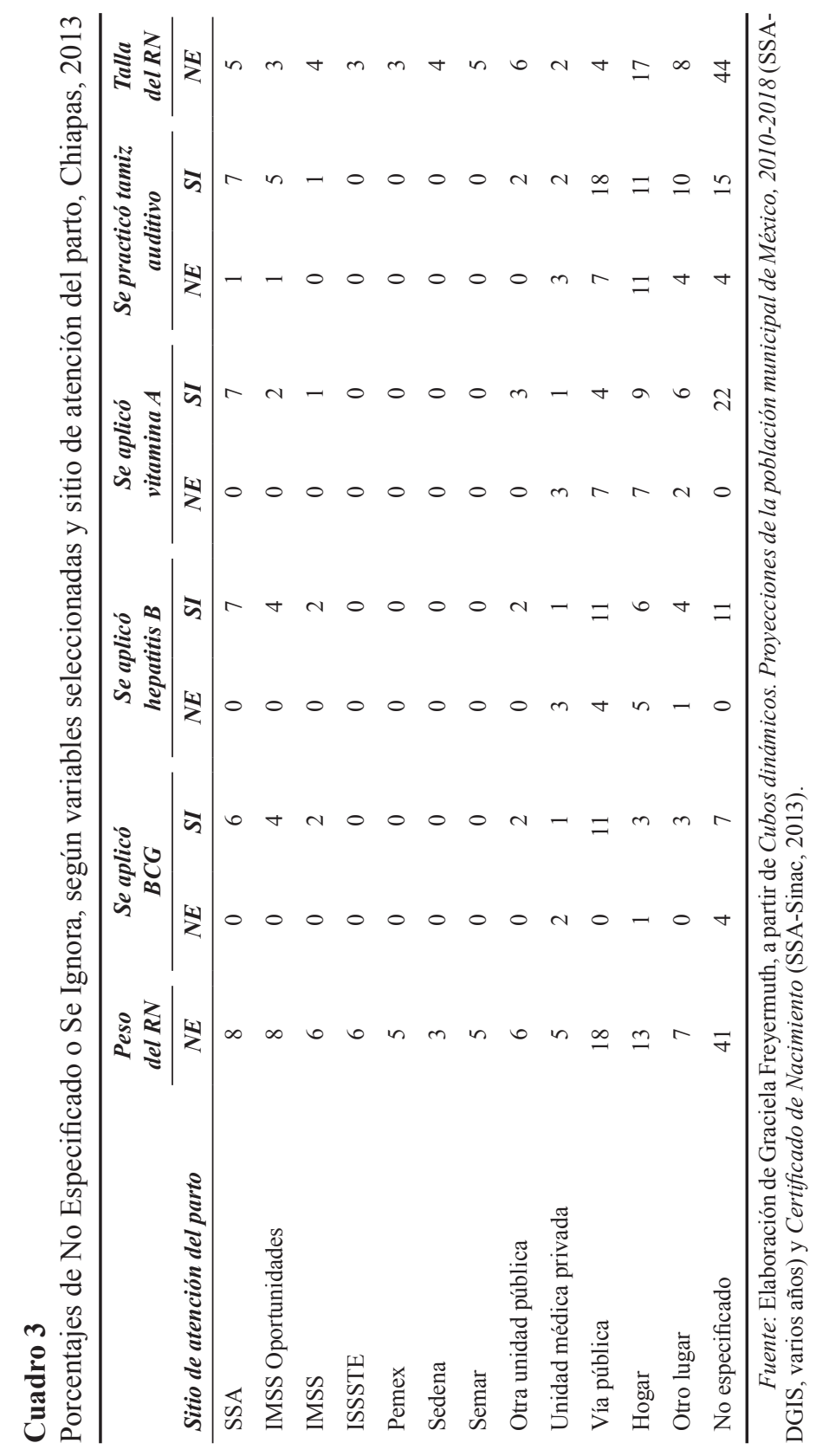




\section{Expedición tardía del Certificado de Nacimiento}

En la NOM 035 SSa3 2012 (NOM 035) se señala que:

11.7. El Certificado de Nacimiento debe expedirse según las circunstancias que acompañen al nacimiento de conformidad con lo siguiente:

11.7.1. Si el nacimiento ocurrió en una unidad médica del SNS, el Certificado de Nacimiento debe ser expedido obligatoriamente dentro de la unidad en las primeras veinticuatro horas después de ocurrido el nacimiento, por el médico que atendió al nacido vivo después del parto. En este caso el Certificado de Nacimiento debe ser entregado a la madre (o quien corresponda a falta de ésta) al egreso de la misma o antes de transcurridas veinticuatro horas después de ocurrido el nacimiento, lo que ocurra primero $[D O F, 2012: 16]$.

Sin embargo, en el Cuadro 4 se puede reconocer que la expedición del certificado después de dos días del nacimiento varía conforme al sitio de atención del parto. Petróleos Mexicanos (Pemex) tiene sólo el 1\% de registrados después de dos días, en cambio en instituciones como el IMSSOportunidades, el Instituto de Seguridad y Servicios Sociales de los Trabajadores del Estado (ISSSTE) y la Secretaría de Marina Armada de México (Semar), dicha proporción es de alrededor de 35\%. Consideración aparte requieren los casos acontecidos en la vía pública, cuyo porcentaje es similar al de las instituciones mencionadas (35\%). Y los acontecidos en el hogar, donde casi la totalidad de $\mathrm{CN}$ se fecharon después de dos días, con un $84 \%$. Una situación semejante existe para los acontecidos en otro lugar, con 52 por ciento.

\section{Trabajo de campo}

\section{Entrevistas en hospitales: llenado del CN}

El análisis de los procesos de expedición del certificado se centra en el trabajo de campo realizado en Los Altos, cuyos municipios están señalados en el Cuadro 1. Aunque la NOM 035 señala que la expedición del CN debe ser realizada por el médico que atendió al nacido vivo después del parto, en los hospitales de la Jurisdicción II de Chiapas la responsabilidad del llenado de este instrumento recae en el personal administrativo. Para ello, el personal responsable se auxilia de las hojas de egresos hospitalarios, de la nota de contrarreferencia, de la libreta de parto y de la cartilla de vacunación, ya que 


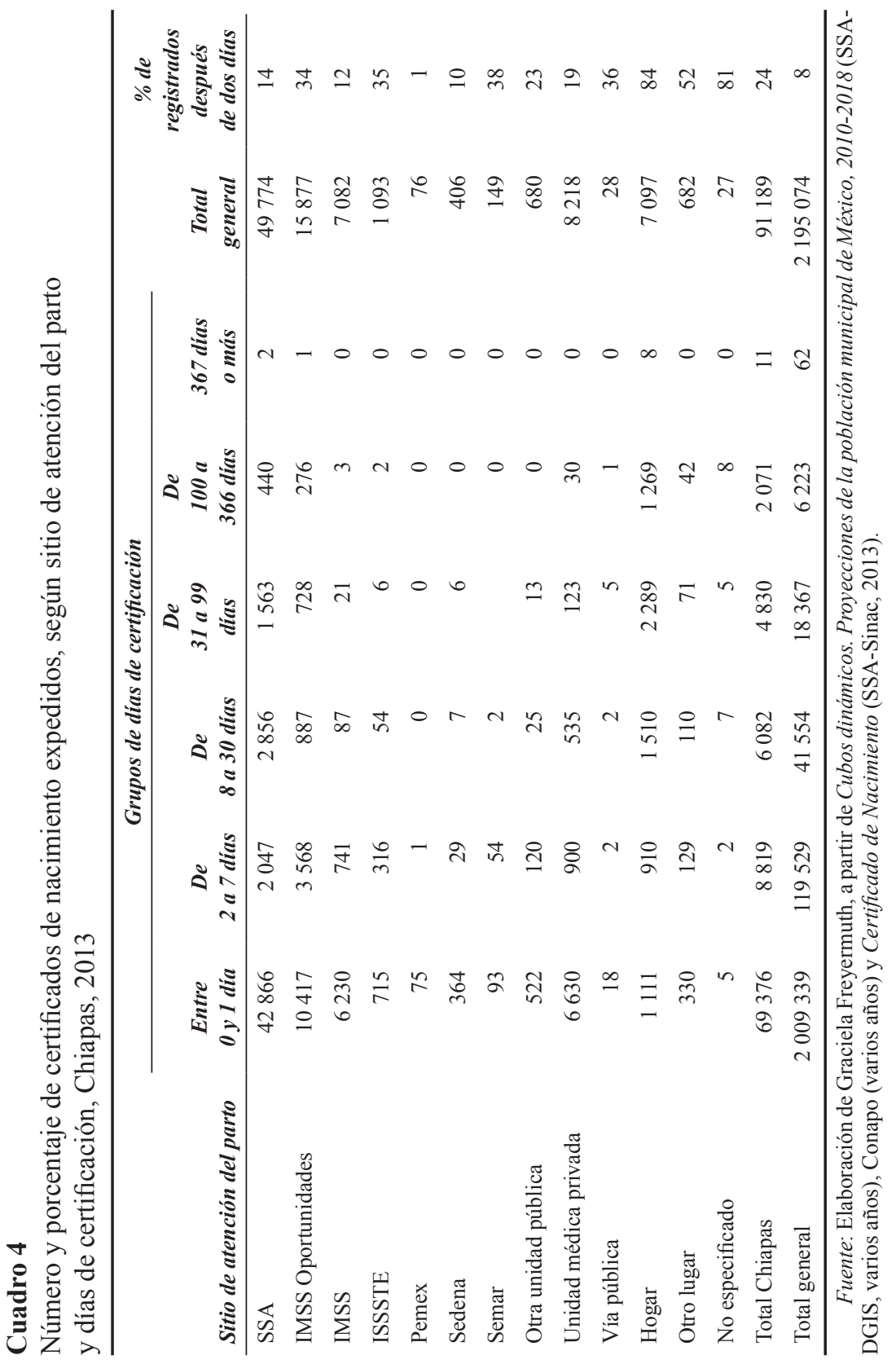


es más sencillo que buscar los datos en el expediente. Los informantes señalaron que frecuentemente los expedientes están incompletos.

Cuando no es posible usar el expediente clínico, el personal administrativo debe solicitar a través de enfermería que le presten la libreta de partos, ya que no puede ingresar a la sala de expulsión. Este personal no está en contacto con las madres, así que debe asociarse con el personal de enfermería o de trabajo social para cumplir su tarea. No hay documentos que aseguren que la información requerida en el certificado esté registrada; por ejemplo, en la Hoja de Egresos Hospitalarios y la Nota de Contrarreferencia, como se señaló, no están incluidos los ítems sobre hijos muertos o hijos vivos, orden de nacimiento, valoración Silverman, ni aplicación de vacunas y vitaminas.

Un entrevistado señaló que lo ideal es que, cuando egresen, los RN se lleven el certificado completo, pero frecuentemente "las parturientas carecen de documentación para confirmar sus datos". De acuerdo con la NOM 035:

11.6. El Certificado de Nacimiento debe ser expedido por única vez a todo nacido vivo en territorio nacional (independientemente de la nacionalidad o situación legal de los padres), en forma gratuita y obligatoria, por un médico con cédula profesional o por la persona facultada por la autoridad sanitaria correspondiente.

Antes de su expedición es indispensable que el certificante haya corroborado el nacimiento, el vínculo madre-hijo/a y verificado la identidad de la madre mediante una identificación oficial, o a falta de esta última, con el documento respectivo expedido por la autoridad competente [DOF, 2012: 16].

En los municipios de Los Altos un porcentaje importante de mujeres que se atienden en el hospital no habita en la cabecera de los municipios donde se ubica el centro de atención y no llevan consigo su identificación oficial. De acuerdo con información del Sinac-2012, en Chiapas $80 \%$ de los partos y $78 \%$ de las cesáreas tienen una permanencia de 24 horas o menos, por ello se dan casos en que los RN egresan $\sin \mathrm{CN}$.

La documentación que se solicita para la expedición del $\mathrm{CN}$ en cada unidad de salud es diversa; por ejemplo, en el municipio de Larráinzar se requiere la Clave Única de Registro de Población (CURP), acta de nacimiento de la madre, credencial del Instituto Nacional Electoral (INE) y Seguro Popular (si se carece de credencial, se puede solicitar una carta de identidad en la presidencia municipal).

En el hospital de Larráinzar señalaron que de aproximadamente $300 \mathrm{CN}$ que expidieron en 2014 , solamente $10 \%$ correspondió a RN que tuvieron parto hospitalario. Aunado a esto, el personal encargado de expedir estos 
certificados no recibe capacitación formal, la capacitación es en cascada y generalmente la realizan los directores de las unidades. Asimismo, el llenado de la plataforma se considera muy sencillo, ya que el que lleva a cabo esa acción en uno de los hospitales visitados comentó que el responsable del Sinac en la jurisdicción le explicó todo el proceso "en cinco minutos".

\section{Estrategia para que los niños que nacen con ayuda de parteras o en el hogar puedan obtener un certificado}

Como señaló el entrevistado en Larráinzar, un porcentaje importante de los partos es atendido por parteras, por ello se requiere establecer mecanismos que faciliten la expedición de los CN. En los casos de Las Rosas, Larráinzar y Aldama se han utilizado estrategias similares. En el Cuadro 1 podemos reconocer que, a diferencia del resto de municipios mayoritariamente indígenas de Los Altos, en Larráinzar y Aldama hay una diferencia relativamente pequeña de los nacidos certificados por el Sinac y los estimados por el Conapo. También se reconoce que la región Los Altos exhibe porcentajes bajos de NE y SI, dado que ahí es muy alto el no registro de nacimientos y la mayoría de los partos ocurre en casa, por lo que su problema principal es precisamente el no registro.

Por otro lado, Larráinzar tiene una Casa de Partos, y alrededor de 46 de las parteras llamadas tradicionales vinculadas con el Hospital Básico Comunitario, están adscritas a la Casa de Partos. Estas parteras periódicamente están en contacto con el Sector Salud a través de talleres y se les dota de una libreta en la cual registran la talla, el peso y la fecha de nacimiento de cada infante. Una situación similar ocurre en Las Rosas, donde señalaron que todas las parteras están dadas de alta en los servicios de salud. Por su parte, en Aldama se ha establecido contacto estrecho con las parteras. Es a través de ellas que las mujeres solicitan su $\mathrm{CN}$ a las unidades de salud.

Las mujeres que no se atienden en el hospital deben acudir espontáneamente por su CN. El ISECH tiene varias estrategias para fomentar el registro en algunos de los municipios: utilizan a las parteras como medio de comunicación con las familias de los RN; se les informa que "es necesario que vengan los señores a sacar su certificado para poder registrar a los recién nacidos". Por otro lado, se lleva un control en los cuadernos de las parteras sobre cuántos partos atienden y se les pide que canalicen a las mujeres, ya sea al centro de salud o al hospital comunitario. Otra estrategia utilizada es el control de embarazadas a través de Prospera (antes Oportunidades), donde dan seguimiento a estas mujeres, y cuando regresan a sus pláticas o 
consultas, les informan sobre los requisitos para que se les otorgue el CN. En los centros de salud donde hay director o directora, son ellos los encargados de llenar los certificados, y también, a través de las parteras, de ubicar a los RN para promover la certificación.

Las comunidades más retiradas son las que tienen mayor subregistro porque son atendidas por pasantes $\mathrm{y}$, en el mejor de los casos, cuentan con una enfermera auxiliar de base. Generalmente es este personal el encargado de expedir los certificados. Aquí la disponibilidad del formato del $\mathrm{CN}$ es el obstáculo para un registro oportuno, ya que tienen que elaborar un oficio y acudir a la jurisdicción para obtener de manera canjeada los certificados. En un centro de salud en Mitontic, la enfermera que era responsable de los certificados señaló que sólo se expiden cuando lo solicitan de manera espontánea, lo que nunca ocurre en el primer año de vida y que prácticamente no ha contado con certificados este año. Esta enfermera no consideraba dicha actividad como parte de su responsabilidad, lo cual evidencia un problema de planeación por parte de la jurisdicción, ya que debería conocer cuántos RN se esperan por unidad de atención. Un informante nos decía: "He conocido niños de seis o siete años que no sólo no están certificados, sino que no tienen vacunas".

Santiago el Pinar es el municipio con la mayor diferencia en las cifras de nacimientos entre las fuentes de información comparadas; cuenta con un Centro de Salud con Servicios Ampliados, sin embargo, durante las visitas a este centro se pudo constatar que prácticamente no tiene demanda de la población, ni estrategia particular para vincularse con las parteras. Señalaron que no expiden $\mathrm{CN}$ a mujeres que no se han atendido en esa unidad de salud.

En la ciudad de San Cristóbal las parteras tienen un formato que deben llenar y proporcionar a las mujeres a quienes atienden. Si no es una partera registrada, entonces los propios familiares deben llenar el formato, que puede ser avalado por el representante de la colonia.

A fin de asegurar la calidad de la información, el $\mathrm{CN}$ debe expedirse según las circunstancias que acompañen al nacimiento:

Para nacimientos atendidos por personal no autorizado para la certificación, las unidades médicas de las instituciones del SNS son las responsables de expedir el certificado correspondiente, dentro de un máximo de 365 días naturales después de ocurrido el nacimiento [SSA-DGIS, 2010: 25].

Este lineamiento es el que debe regir a gran parte de los partos de Los Altos y muy probablemente en otras regiones indígenas del país, ya que no son atendidos por un médico y ni siquiera por una partera certificada. 
Dado que el objetivo del $\mathrm{CN}$ es promover el registro oportuno, homogéneo, veraz e íntegro de los nacidos vivos ocurridos en el país, este documento debe ser expedido para todo nacido vivo en territorio mexicano, independientemente de la nacionalidad o situación legal de los padres. El único requisito para la expedición del certificado es la identificación oficial de la madre o una constancia de identidad emitida por la autoridad correspondiente (municipal, ejidal, de la colonia o barrio).

Sin embargo, cada unidad tiene sus propios criterios y los requisitos aumentan en la medida en que la localidad incrementa su población. En Larráinzar se requiere credencial de elector o carta de identidad, copia del acta de nacimiento de la madre y el acompañamiento de la partera. En Las Rosas exigen acta de nacimiento, credencial de elector, comprobante de domicilio y cartilla de vacunación (con una copia de cada documento), así como credencial de la partera que está certificada. Al preguntar qué hacen cuando una mujer no es atendida por una partera o una partera certificada, señalaron que nunca les ha ocurrido. En San Cristóbal de Las Casas adicionalmente solicitan la credencial de elector de la partera y copia del alta cuando se tiene Seguro Popular, constancia de alumbramiento de la partera $\mathrm{y}$, tratándose de menores de seis meses, deben acudir la partera, el niño y la madre para hacer el trámite; en algunos casos solicitan que la partera esté certificada por el ISECH u otra institución. Adicionalmente, se señala que solamente se expedirán certificados para niños menores de seis meses. En otra unidad médica de San Cristóbal indican que el acta de nacimiento de la madre debe haber sido solicitada en 2009 o posteriormente (es decir, actualizada), copia de credencial de elector vigente, y si es menor de edad solicitan una carta de identidad expedida por la presidencia municipal con fotografía, copia de la CURP, constancia de nacimiento emitida por la partera (con su nombre, domicilio, nombre de la madre, domicilio donde se llevó a cabo el parto, sexo del bebé, peso al nacimiento, cuánto midió y hora de nacimiento), copia del Seguro Popular, ISSSTE o IMSS, comprobante de domicilio de dos meses de antigüedad como máximo, copia vigente de credencial de elector de la partera, y acudir al centro de salud de manera obligatoria la madre, el RN y la partera. Si la partera no está registrada en los servicios de salud, deberá llevar constancia del representante de la colonia acerca de cuántos años lleva ejerciendo como partera empírica. 


\section{Entrevistas en el Registro Civil}

Tradicionalmente los responsables de los RC han venido expidiendo actas de nacimiento sin CN. A partir de 2014 tienen el mandato de no emitirlas sin el respectivo CN. Sin embargo, en Los Altos, aunque todos los oficiales del $\mathrm{RC}$ están solicitando los $\mathrm{CN}$, este requisito no lo consideran obligatorio todavía. El oficial del RC de Chamula señaló "Aquí nada es obligatorio". En los casos de San Juan Chamula, Chalchihuitán, Pantelhó, Chenalhó, Aldama, Cancuc, Mitontic, Larráinzar, Altamirano, Oxchuc, Huixtán, Chanal y Zinacantán, por acuerdo de las autoridades municipales se seguirán expidiendo actas de nacimiento sin $\mathrm{CN}$.

Como señaló con preocupación un oficial de RC de la región:

Nos están pidiendo que la familia traiga a la partera y que juntos vayan al centro de salud para expedir el certificado. Esto es especialmente complicado porque en Chamula los partos los atiende el esposo, la tía, la hermana o la abuelita, y en aquellos casos en que los atiende la partera no será posible que la familia traiga a la partera por los gastos que esto representa [Oficial de RC, 11 de septiembre, 2014].

La mayor parte de los oficiales del RC considera a este evento como el de mayor importancia, con excepción del oficial de Chalchihuitán, quien señaló que era el matrimonio. Prácticamente todos mencionaron que el acta de nacimiento responde al derecho de contar con una identidad.

En los últimos años se ha exentado el pago del registro de nacimiento: hace dos años se exentaba a los menores de seis meses; en 2014 a los menores de un año; en 2015 a los menores de 18 años. Cuando se expide el acta de nacimiento en el mismo día, también se exenta el pago de la copia impresa.

Los requisitos para el registro del acta de nacimiento son para los menores de un año y se requiere credencial de elector, si no se cuenta con ella pueden presentar una constancia de identidad con fotografía o no, dependiendo del municipio; el aviso de nacimiento, denominado constancia de alumbramiento por parte de las parteras, o el CN. Existe un formato que contiene el nombre de la madre, su lugar de origen y domicilio; en el caso del RN: sexo, fecha y hora de nacimiento. En otros formatos se pide el nombre de los dos padres. Dicho formato puede ser llenado por la partera o el agente municipal, pero requiere la firma o la huella digital de la partera.

En aquellos casos en que la atención del parto se haya realizado por un familiar u otra persona que no está reconocida como partera, se realiza un escrito en el que se señala que será la única vez que por razones circunstan- 
ciales esa persona ha atendido el parto, y que de otra manera tendría que darse de alta como partera en los servicios de salud.

En Altamirano se tiene un padrón de parteras en el RC; en el caso de Oxchuc es la presidencia quien lo regula. Este padrón lo utilizan para capacitar a las parteras y advertirles que no pueden expedir constancias de alumbramiento cuando no atendieron el parto porque pueden ser sancionadas, es decir, pueden ser consignadas. Cuando un nacimiento lleva el nombre de una partera que no está en el padrón, entonces se le cita en la oficialía para contar con toda la información que la identifique. Todas las constancias deben estar avaladas por una autoridad local.

El acompañamiento de la partera no es un requisito al que se hayan referido en todos los casos; solamente en uno señalaron que, si no había identificación oficial, bastaba con un certificado de identidad expedido por la presidencia municipal del lugar. De acuerdo con las entrevistas, todavía es muy irregular la disponibilidad de los $\mathrm{CN}$. Adicionalmente señalaron que frecuentemente en las unidades de salud no está presente el personal que debe llenar los $\mathrm{CN}$; por ello todas estas oficialías seguirán expidiendo actas sin los CN, al margen de lo que marca la NOM 035.

En la mayoría de las oficialías se señaló el incremento en la incidencia de registros de menores de un año, lo que adjudican a que ya es un trámite gratuito y al interés de los padres, dado que en algunos programas como Prospera, Seguro Popular o en los apoyos para madres solteras, se les solicita el acta de nacimiento de los hijos para darles los servicios. En Oxchuc, el oficial mencionó que esto también ha producido un interés de la pareja por tener más hijos. En cuanto a la gratuidad del registro, el oficial de Altamirano indica que también "muchos se confían y esperan hasta un día antes de cumplir los 18 años para ir a registrarlos". En varios municipios persiste el registro de niños y niñas de 12 y 13 años.

En Chenalhó y Altamirano aseguraron que acuden muchos exzapatistas a registrar hijos adolescentes, dado que ya se están deslindando de este movimiento. En Chamula mencionaron que se observa interés en registrar, pero que, a pesar de ser gratuito, las personas no acuden por la lejanía y la falta de dinero para el transporte.

Las campañas que se ofrecen en las comunidades han sido de gran utilidad para sensibilizar a las personas sobre el pronto registro, o para extemporáneos y adultos mayores. En algunos municipios se sigue haciendo promoción por parte de los oficiales que van a registrar o por medio de comunicación informal.

En la mayoría de las oficialías indican que para el registro es primordial que asistan padre y madre, pero en caso de que sólo acuda la madre es posible 
hacer el registro en el momento. Si únicamente acude el padre, para poder proceder éste tiene que traer un oficio de la procuraduría del Sistema Nacional para el Desarrollo Integral de la Familia (DIF) que acredite la paternidad.

En cuanto a la captura de los registros, los oficiales y auxiliares mencionaron que existen errores a la hora de que las personas llevan sus $\mathrm{CN}$ para registrar a sus hijos. La mayoría de éstos consiste en la modificación de los datos de la madre y de los datos del RN, como hora de nacimiento, lugar y sexo. En Chamula, Chenalhó, Oxchuc y Pantelhó informaron que reciben capacitaciones cada mes sobre reglamentos del RC.

\section{Consideraciones finales}

El objetivo del CN no sólo es generar información homogénea de los nacimientos, sino que ésta sea oportuna para lograr la eficiencia a la hora de calcular la tasa de mortalidad infantil, la RMM y los indicadores de fecundidad nacionales, y que se otorgue acta de nacimiento a niños y niñas para que accedan a sus derechos esenciales. Las dificultades de la oportunidad en el registro de nacimientos y los problemas que esto conlleva en la planeación de los programas de salud y sociales ya han sido evidenciados (Aguirre y Camposortega, 1980; Figueroa, 2009; Welti, 2009; García, 2016).

Un estudio realizado entre 2007 y 2008 (Hernández y cols., 2012) mostró que solamente $31.89 \%$ de los menores contaba con certificado de nacimiento, pero sólo $8.32 \%$ contaba con certificado y con acta, y $73.9 \%$ de los recién nacidos que tenía certificado no tenía acta. De igual manera, 62.8\% de los nacimientos que no tenían certificado de nacimiento tampoco tenían acta. Por lo que el CN, a mediano plazo, podría ser un instrumento que permita contar con el número de nacimientos del país de manera oportuna, independientemente de la solicitud temprana de actas de nacimiento. Algunas de las conclusiones a las que llega el estudio de Hernández y cols. (2012) es que la ausencia del $\mathrm{CN}$ responde a que los nacimientos ocurrieron entre mujeres hablantes de lengua indígena, que las mujeres no contaban con Seguro Popular, y que el nacimiento ocurrió con partera.

En Chiapas, actualmente se ha observado una mejora en el conocimiento del número de nacimientos a partir del $\mathrm{CN}$, lo cual ha sido a partir de que una gran mayoría de los partos de 2015 en la entidad (91.7\%), según información oficial (Gobierno de la República, México, 2017), fueron atendidos por personal sanitario capacitado. Por otro lado, en la encuesta intercensal se muestra que $80.2 \%$ de la población indígena en ese estado cuenta con Seguro Popular (Luna y Freyermuth, 2017). Sin embargo, un estudio a nivel 
nacional sobre las necesidades de las usuarias en la atención materna, mostró que $85 \%$ de las mujeres atendidas con parteras tradicionales o en su casa por algún familiar, contaban con Seguro Popular (Freyermuth, 2017). En concordancia con estos hallazgos, la encuesta de opinión realizada por el Comité Promotor por una Maternidad Voluntaria y Segura en Chiapas (2016), relacionada con las preferencias de las mujeres durante la atención del parto, mostró que $75 \%$ de las mujeres prefería la atención con parteras o en su casa con un familiar. Por lo tanto, aun cuando se ha incrementado la atención del parto de las mujeres chiapanecas por personal capacitado, las parteras aún tienen un gran peso.

Uno de los reclamos de las parteras llamadas tradicionales y de las parteras profesionales se refiere a los problemas que están enfrentando las madres que se atienden con ellas para la obtención del $\mathrm{CN}$ y por tanto del acta de nacimiento. ${ }^{6}$ Estos hallazgos deben ser tomados en consideración a fin de que se faciliten, en estos casos, los trámites de obtención del CN y, por tanto, del acta de nacimiento.

Se ha establecido que la expedición del $\mathrm{CN}$ se realice en los servicios de salud, con el menor número de trámites, inclusive cuando los partos ocurran al margen del SNS. Sin embargo, por lo menos se están solicitando de dos a tres documentos, y en la ciudad de San Cristóbal solicitan hasta diez requisitos distintos, lo que lleva a que se pierda la esencia de la certificación y su oportunidad. Es muy importante asegurar el objetivo de la NOM 035, en cuanto a que quien expida el CN pueda certificar el vínculo madre-hijo y la identidad de la madre; éstos serían los requisitos fundamentales para la expedición del CN.

Aun cuando desde 2007 se estableció el CN como requisito obligatorio para hacer el registro y obtener el acta de nacimiento en el RC, no está considerado de esta forma en la última actualización del Reglamento del RC de Chiapas (P.O. 28 diciembre, 2011). No se menciona explícitamente el CN como requisito, sino que se enuncia como una "constancia de parto, expedida por hospital público, privado o partera(o) empírica(o)". ${ }^{7}$ Se recomienda realizar mayores esfuerzos para incidir en las modificaciones de los reglamentos con el propósito de que estén armonizados con las distintas disposiciones de la SSA y se pueda cumplir con los objetivos que se proponen.

En cuanto al análisis específico de los ítems del CN, en este estudio se encontró que los datos relacionados con la historia reproductiva de la madre

6 Senado de la República, Comisión de Salud, Primer Foro por el Derecho a la Partería en México, Salón de la Comisión Permanente, Mesa del Certificado de Nacimiento al Derecho a la Identidad, 14 de marzo 2017.

7 Artículo 47, fracción II, Reglamento del Registro Civil del Estado de Chiapas, 2011. 
y algunos datos del nacido vivo y del nacimiento, particularmente aquellos relacionados con la valoración y los cuidados del $\mathrm{RN}$, tenían gran cantidad de SI o NE. Por lo tanto, es necesario mejorar la estrategia de acopio de información en los registros civiles, para que cuando se haga, sea de manera efectiva. Por ejemplo, se ve necesario contar con un informante adecuado con interés y capacitado, formular las preguntas acordes a la población que se está entrevistando y, de preferencia, procurar estar en contacto con la madre en el momento de llenar el formato.

Para reducir errores y validar la información capturada, hay que llevar a cabo algunas modificaciones al $\mathrm{CN}$, como indicar el número de años acumulados de escolaridad en lugar del nivel y grado; incluir la variable número de abortos para validar los referentes a número de embarazos, hijos vivos y muertos; en el apartado "Datos de la madre" invertir las variables 13, 14 y 15 por las 6,7 y 8 , ya que las últimas se refieren a datos sociodemográficos. Además pueden utilizarse las siguientes dos variables también como validación: orden de nacimiento y sobrevivencia del hijo anterior, dado que tendrían que estar relacionadas, identificándose en el sistema cuando haya error.

Algo que llama la atención es que la ausencia de respuesta no es exclusiva de los partos atendidos al margen de los sistemas de salud, lo que puede obedecer a que las personas encargadas del llenado de los certificados en el ISECH no están forzosamente en contacto con las madres y se nutren, para su expedición, de documentos que no necesariamente registran toda la información que se requiere para el certificado.

Como en otras situaciones, las mujeres más pobres, las que se atienden al margen de los servicios de salud, las que cuentan con menos recursos para sacar copias y actualizar documentos, son las que deben realizar más trámites, lo que necesariamente provocará problemas de subregistro en Chiapas y particularmente en las regiones indígenas.

\section{Bibliografía}

Aguirre, Alejandro y Sergio Camposortega (1980), "Evaluación de la información básica sobre mortalidad infantil en México", Demografia y Economía, vol. 14, núm. 4, pp. 447-466. Disponible en: http://estudiosdemograficosyurbanos. colmex.mx/index.php/edu/article/view/470/463

Bobadilla, José y cols. (1987), "Cobertura y calidad del registro de defunciones perinatales ocurridas en instituciones de salud del Distrito Federal", Estudios Demográficos y Urbanos, vol. 2, núm. 2 (5), pp. 257-271. Disponible en: http:// estudiosdemograficosyurbanos.colmex.mx/index.php/edu/article/view/628/621 
Camposortega, Sergio (1992), Análisis demográfico de la mortalidad en México 1940-1980, México, El Colegio de México, A.C.

CEPAL (2011), Guía para asegurar la calidad de los datos censales, Santiago de Chile, Comisión Económica para América Latina y el Caribe, Naciones Unidas (Manuales). En línea: http://www.cepal.org/es/publicaciones/5515-guia-asegurarla-calidad-datos-censales

CEPAL (2014), Los datos demográficos: alcances, limitaciones y métodos de evaluación, Santiago de Chile, Comisión Económica para América Latina y el Caribe (Manuales), pp. 65-86. Disponible en línea: http://repositorio.cepal.org/ handle/11362/37145

Colón, Francisco y cols. (1998), "Mortalidad perinatal en un municipio de Quintana Roo, México, en 1995 y 1996", Revista Mexicana de Pediatría, vol. 65, núm. 6, pp. 251-253. Disponible en: http://www.medigraphic.com/pdfs/pediat/sp-1998/ sp986f.pdf

Comité Promotor de una Maternidad Segura y Voluntaria en Chiapas (2016), “El desmantelamiento de la atención obstétrica en el primer nivel de atención, como determinante de la violencia obstétrica y de género contra las mujeres indígenas de Chiapas", amicus curiae presentado al Tribunal Simbólico Sobre Violencia Obstétrica en México, Ciudad de México, 5 de mayo.

Conapo (varios años), Proyecciones de la población 2010-20150, México, Consejo Nacional de Población. Disponible en: www.conapo.gob.mx/es/CONAPO/ Proyecciones

De León, Lourdes (1999), "Verbs roots and caregiver speech in early Tzotzil (Mayan) acquisition", en Barbara Fox, Dan Jurafsky y Laura A. Michaelis (coords.), Cognition and function in language, Stanford, California, Publications Center for the Study of Language and Information.

DOF (2010), "Reglamento interior de la Secretaría de Salud", Diario Oficial de la Federación, México, Secretaría de Gobernación. Disponible en: http://www. geriatria.salud.gob.mx/descargas/administracion/reglamento_interior_SS.pdf (2 de febrero de 2015).

DOF (2012), "Norma Oficial Mexicana NOM035SSa32012 en materia de información en salud", Diario Oficial de la Federación, México, Secretaría de Gobernación. Disponible en: http://dof.gob.mx/nota_detalle.php?codigo=5280848\& fecha $=30 / 11 / 2012$ (28 de julio de 2015).

Espíritu, Nora y cols. (2007), "Discrepancias en el registro de la mortalidad perinatal en Lima y Callao según fuente de información", Revista Peruana de Medicina Experimental y Salud Pública, vol. 24, núm. 4, pp. 363-69. Disponible en: http:// www.rpmesp.ins.gob.pe/index.php/rpmesp/article/view/1135

Figueroa, Beatriz (2009), "El registro de los nacimientos en el Distrito Federal: características y problemas de la información derivada de estos registros", en Luz María Valdés (coord.), Conmemoración del 150 aniversario del Registro Civil. Fundamentos y reflexiones, México, Universidad Nacional Autónoma de México. 
Freyermuth, Graciela (2017), "La partería en México desde el punto de vista de las usuarias", trabajo presentado en el Primer Foro por el Derecho a la Partería en México, Comisión de Salud, Chiapas, 14 de marzo.

Freyermuth, Graciela y Rosario Cárdenas (2009), "Evaluación del subregistro de la mortalidad materna en Los Altos de Chiapas mediante las estrategias RAMOS y RAMOS modificada", Salud Pública de México, vol. 51, núm. 6. Disponible en: http://www.scielo.org.mx/pdf/spm/v51n6/a02v51n6.pdf

Freyermuth, Graciela, Marisol Luna y José A. Muños (2015), Indicadores 2013. Objetivo de Desarrollo del Milenio 5: Avances en México, México, Centro de Investigaciones y Estudios Superiores en Antropología Social (CIESAS) / Observatorio de Mortalidad Materna en México (OMM) / Organización Panamericana de la Salud en México (OPS).

García, Juan (2016), "La cobertura oportuna y subcobertura de los nacimientos en México", Coyuntura Demográfica, núm. 10. Disponible en: http://www.somede. org/coyuntura-demografica/pdf/numero10/garcia.pdf

Gobierno de la República, México (2017), Objetivos de Desarrollo del Milenio, 5. Mejorar la Salud Materna, México, Secretaría de Gobernación. Disponible en: http://www.objetivosdedesarrollodelmilenio.org.mx/cgi-win/odmsql.exe/INDO DM005000100020,27,0,000,False,False,False,False,False,False,False,0,0,E (21 de marzo, 2017).

González, Alfonso y Rosario Cárdenas (2005), "Una aproximación a la medición del subregistro de nacimientos en las estadísticas vitales de México", Estudios Demográficos y Urbanos, vol. 20, núm. 3 (60), pp. 619-625. Disponible en: http://estudiosdemograficosyurbanos.colmex.mx/index.php/edu/article/ view/1212

Guiteras, Calixta (1992), Cancuc: etnografia de un pueblo tseltal de Los Altos de Chiapas, 1944, $1^{\text {a }}$ edición, Chiapas, Gobierno del Estado de Chiapas, Consejo Estatal para el Fomento a la Investigación y Difusión de la Cultura (Nuestros Pueblos).

Hernández, Bernardo y cols. (2012), "Subregistro de defunciones de menores y certificación de nacimiento en una muestra representativa de los 101 municipios con más bajo índice de desarrollo humano en México", Salud Pública de México, vol. 54, núm 4. Disponible en: http://www.scielo.org.mx/pdf/spm/ v54n4/ 09.pdf

INEGI (2003), Sintesis metodológica de las estadísticas vitales, México, Instituto Nacional de Estadística y Geografía.

INEGI (2010), Censo de Población y Vivienda 2010, México Instituto Nacional de Estadística y Geografía. Disponible en: http://www.inegi.org.mx/est/lista_cubos/ consulta.aspx?p=pob\&c=1 (22 de mayo de 2015).

Lozano, Rafael (2008), "¿Es posible seguir mejorando en los registros de las defunciones en México?", Gaceta Médica Mexicana, vol. 144, núm. 6, pp. 525-533. Disponible en: http://www.anmm.org.mx/GMM/2008/n6/59_vol_144_n6.pdf Lozano, R. et al. (2005), "Medición de la mala clasificación de la mortalidad mater- 
na en México, 2002-2004”, Sintesis Ejecutiva, núm. 16. Disponible en: http:// www.sintesisejecutiva.com.mx/ (15 de enero de 2017).

Luna, Marisol y Graciela Freyermuth (2017), "Población hablante de lengua indígena en México: indicadores sociodemográficos 2015", documento, Ciudad de México, Centro de Investigaciones y Estudios Superiores en Antropología Social. Disponible en el blog del Observatorio de Mortalidad Materna en México: http:// www.omm.org.mx/index.php/blog/230-poblacion-hablante-de-lengua-indigenaen-mexico-indicadores-sociodemograficos-2015

Ordorica, Manuel y cols. (1975), "Evaluación de la mortalidad infantil en la República Mexicana, 1930-1970”, Evaluación y Análisis, vol. 3, núm. 4, México, Dirección General de Estadística.

Rodríguez-Angulo, Elsa y cols. (2009), "Subregistro de muertes maternas en comunidades mayas del oriente de Yucatán", Revista Biomédica, vol. 20, núm. 2, pp. 90-98. Disponible en: http://revistabiomedica.mx/index.php/revbiomed/ article/view/145

SSA-DGIS (2009), Búsqueda intencionada de muertes maternas en México. Informe 2008, $1^{a}$ edición, México, Secretaría de Salud, Subsecretaría de Integración y Desarrollo del Sector Salud, Dirección General de Información en Salud.

SSA-DGIS (2010), Manual de llenado del Certificado de Nacimiento, México, Secretaría de Salud, Dirección General de Información en Salud, Subsistema de Información sobre Nacimientos.

SSA-DGIS (varios años), Cubos dinámicos. Proyecciones de la población municipal en México, 2010-2018, México, Secretaría de Salud, Dirección General de Información en Salud.

SSA-Sinac (2013), Certificado de Nacimiento, México, Secretaría de Salud, Subsistema de Información sobre Nacimientos.

Suárez, Patricia (1981), "Estimation of under registration of infant deaths in Mexico", tesis de maestría, Londres, London School of Hygiene and Tropical Medicine, Centre for Population Studies.

Tomé, Patricia y cols. (1997), "Características asociadas al subregistro de muerte en niños del estado de Guerrero", Salud Pública de México, vol. 39, núm. 6. Disponible en: http://www.scielosp.org/scielo.php?script=sci_arttext\&pid=S0036$36341997000600005 \& \operatorname{lng}=\mathrm{en} \& \mathrm{nrm}=\mathrm{iso} \& \mathrm{t} \operatorname{lng}=\mathrm{es}$

UNICEF e INEGI (2012), Derecho a la identidad. La cobertura del registro de nacimiento en México en 1999 y 2009, México, Fondo de las Naciones Unidas para la Infancia / Instituto Nacional de Estadística y Geografía.

Valdés, Luz María (2009), Conmemoración del 150 aniversario el Registro Civil. Fundamentos y reflexiones, México, Universidad Nacional Autónoma de México.

Welti, Carlos (2009), "El Registro Civil y el conocimiento de la realidad nacional", en Luz María Valdés (coord.), Conmemoración del 150 aniversario del Registro Civil. Fundamentos y reflexiones, México, Universidad Nacional Autónoma de México. 


\section{Acerca de los autores}

María Graciela Freyermuth Enciso es licenciada en Medicina por la Universidad Nacional Autónoma de México (UNAM), maestra en Medicina Social por la Universidad Autónoma Metropolitana, unidad Xochimilco, y doctora en Antropología Social por la UNAM. Desde 1988 es investigadora titular del Centro de Investigaciones y Estudios Superiores en Antropología Social, unidad Sureste. Sus proyectos se han centrado en la salud reproductiva, la violencia de género, las políticas de salud en un contexto multicultural, la migración, y específicamente en la muerte materna y mortalidad femenina en la región de Los Altos de Chiapas y en el ámbito nacional. Es autora o coautora de diez libros. Cuenta con varias docenas de artículos científicos y de divulgación. Pertenece al Sistema Nacional de Investigadores, nivel II. Fue consejera académica (2006-2010) del Consejo Nacional de Evaluación de la Política de Desarrollo Social, secretaría técnica del Comité Nacional por una Maternidad Sin Riesgos en México (2008-2012), y secretaria técnica del Observatorio de Mortalidad Materna en México (20102016). Ha recibido distinciones académicas nacionales y estatales.

María del Pilar Ochoa Torres es licenciada en Psicología por el Instituto Tecnológico de Sonora (Itson); tiene estudios de maestría en Población y Desarrollo por la Facultad Latinoamericana de Ciencias Sociales (Flacso), y cuenta con un diplomado en Estadística Aplicada de la Facultad de Economía de la Universidad Nacional Autónoma de México (UNAM) y otro en Violencia Familiar y Derechos Humanos, del Instituto de Investigaciones Jurídicas de la UNAM.

Actualmente es asesora en la Subsecretaría de Integración y Desarrollo del Sector Salud. Ha participado en varios proyectos del Centro de Investigaciones y Estudios Superiores en Antropología Social (CIESAS), unidades Sureste y D.F., como el Observatorio de Mortalidad Materna (OMM), en donde se desempeñó como analista de información estadística y creación de indicadores principalmente referentes a morbilidad y mortalidad materna, salud sexual y reproductiva, y presupuesto para programas de salud de la mujer. También participó en un proyecto en El Colegio de México, A.C. relativo a un sistema de monitoreo del comportamiento violento de la sociedad contra las mujeres.

José Alberto Muños Hernández es licenciado en Matemáticas, maestro en Demografía y doctor en Ciencias en Salud Pública, con área de concentración en Sistemas de Salud. Actualmente se desempeña como catedrático Conacyt, 
adscrito al CIESAS Pacífico Sur, en donde investiga sobre temas de salud y pueblos indígenas. Ha participado en proyectos como el de "Mercados de trabajo y condiciones de vida de las familias en México: una propuesta de indicadores", El Colegio de México, A.C. Participó como encargado de validación en el área de indicadores en el Observatorio de Mortalidad Materna en México (OMM). Fue responsable de la capacitación a miembros del OMM para el cálculo de indicadores de mortalidad materna utilizando las bases de datos públicas del Sector Salud y los cubos dinámicos de información de la DGIS con Stata. Realizó trabajo de campo en la implementación de una encuesta nacional para la Universidad Nacional Autónoma de México. Cuenta con experiencia profesional como investigador en ciencias médicas nivel A en el Instituto Nacional de Salud Pública sobre diferentes temas como: control de tabaco en México; cálculo de indicadores, análisis y procesamiento de datos de las bases de mortalidad y egresos hospitalarios de la DGIS; y proyección de necesidades y población del Instituto de Seguridad y Servicios Sociales de los Trabajadores del Estado (ISSSTE).

Fecha de recepción: 15 de octubre de 2015.

Fecha de aceptación: 27 de abril de 2017. 